\title{
Instabilités de flambage dans les fluides visqueux \\ Du laboratoire au manteau terrestre
}

Neil M. Ribe ${ }^{1}$ (ribe@ipgp.jussieu.fr), Mehdi Habibi ${ }^{2,3}$, Daniel Bonn ${ }^{3,4}$, Éléonore Stutzmann ${ }^{1}$

1 - Institut de Physique du Globe de Paris et Université de Paris 7, 4 place Jussieu, 75252 Paris Cedex 05.

2 - Institute for Advanced Studies in Basic Sciences, Zanjan 45195-1159, Iran.

3 - Laboratoire de Physique Statistique, École Normale Supérieure, 75231 Paris Cedex 05.

4 - Van der Waals-Zeeman Institute, University of Amsterdam, 1018 XE Amsterdam, Pays-Bas.

Un mince filet de fluide visqueux qui tombe sur une surface s'enroule en hélice tournante, alors que le même fluide en forme de nappe se replie de manière périodique. Ces instabilités, dites " de flambage ", cachent une physique d'une grande complexité, avec notamment quatre régimes qui correspondent aux différents équilibres des forces visqueuse, gravitationnelle et inertielle. La gamme de leurs applications va des industries de l'alimentation et de fabrication des polymères jusqu’à la dynamique du manteau de notre planète.
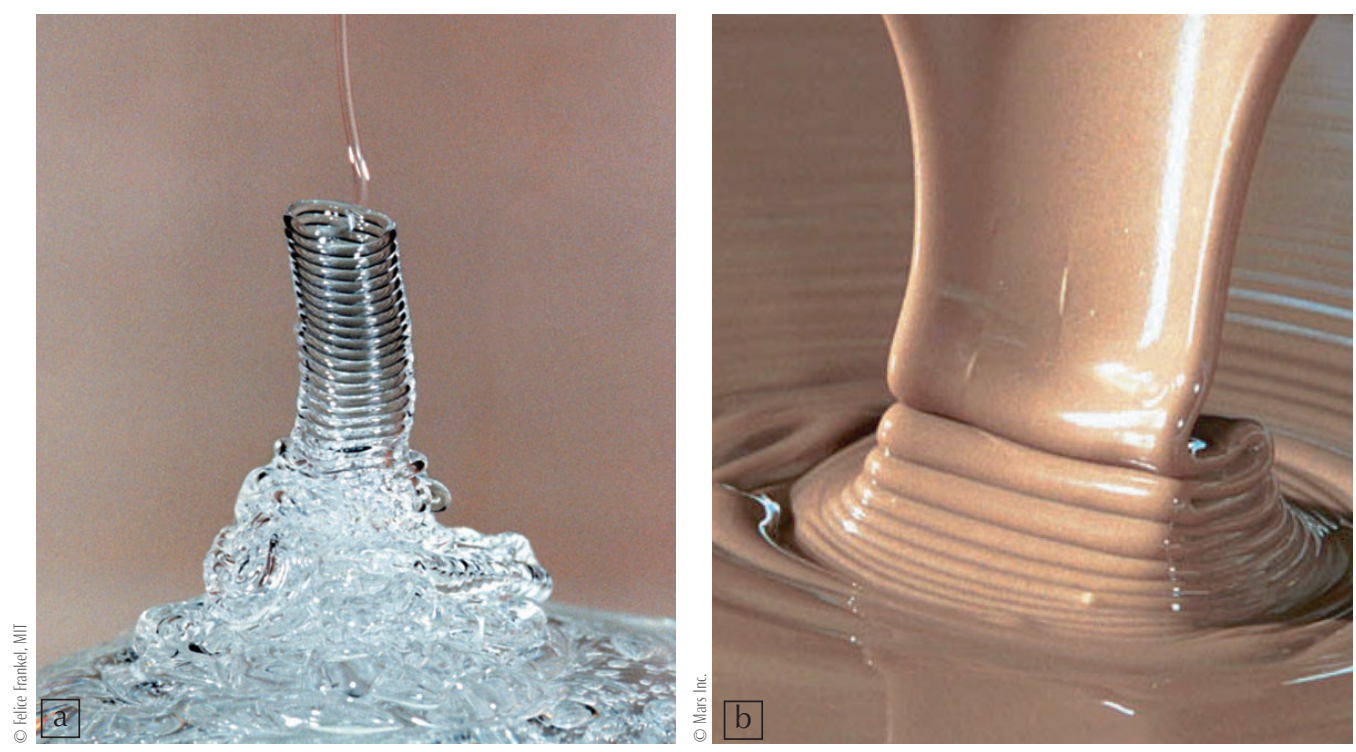

1. Instabilités de flambage dans les fluides visqueux. (a) Enroulement d'un filet d'huile de silicone. (b) Repliement périodique d'une nappe de chocolat fondu.

À votre petit déjeuner demain matin, laissez tomber un mince filet de miel d'une hauteur d'une dizaine de $\mathrm{cm}$ sur une tartine : le filet s'enroulera sur lui-même pour former un petit " tire-bouchon tournant" (fig. 1a). Si vous n'aimez pas le miel, versez sur votre tartine une nappe de chocolat fondu : elle se pliera de manière périodique (fig. 1b). Ce sont deux beaux phénomènes de mécanique des fluides qui se manifestent dans des situations très diverses, du remplissage des boîtes dans l'industrie de l'alimentation jusqu'à la subduction des plaques tectoniques dans le manteau terrestre. Mais la simplicité de leur réalisation cache une physique d'une étonnante complexité, qui attire l'attention des spécialistes depuis une cinquantaine d'années. Pour notre part, nous essayons depuis quelque temps de jeter un nouveau regard sur ces phénomènes, en combinant des approches expérimentale, analytique, numérique et (pour le volet géophysique de l'étude) observationnelle.

L'enroulement et le repliement périodique sont deux exemples des instabilités de flambage, où un objet mince se plie sous l'influence d'une compression axiale [1]. L'exemple type en est une tige élastique en forme de cylindre droit de longueur $H$, diamètre $d$ et module élastique $E$, soumise à une force axiale compressive $P$ (fig. 2a). Lorsque $H>H_{c}=A d^{2}(E / P)^{1 / 2}$ où $A$ est une constante dont la valeur dépend des conditions aux limites aux deux extrémités, la tige devient instable et se plie (fig. 2b). Pour créer un système analogue avec un fluide visqueux de viscosité $v$, densité $\rho$ et capillarité $\gamma$, on le fait extruder à un débit volumétrique constant $Q$ par un trou de diamètre $d$ vers une surface solide à une distance $H$ plus bas (fig. 2 c). . La « tige » de fluide ainsi générée s'épaissit fortement vers le bas à cause de la force compressive axiale que crée l'écrasement du fluide contre la surface. Une analyse de cette situation [2] montre que l'instabilité se manifeste sous forme d'une oscillation flexurale (fig. 2d) lorsque $H$ atteint une valeur critique $H_{c}=B d$, où $B(=3$ à 15) augmente en fonction de $\gamma$, mais ne dépend que faiblement de $v$ et de $Q$. Le résultat est le même 

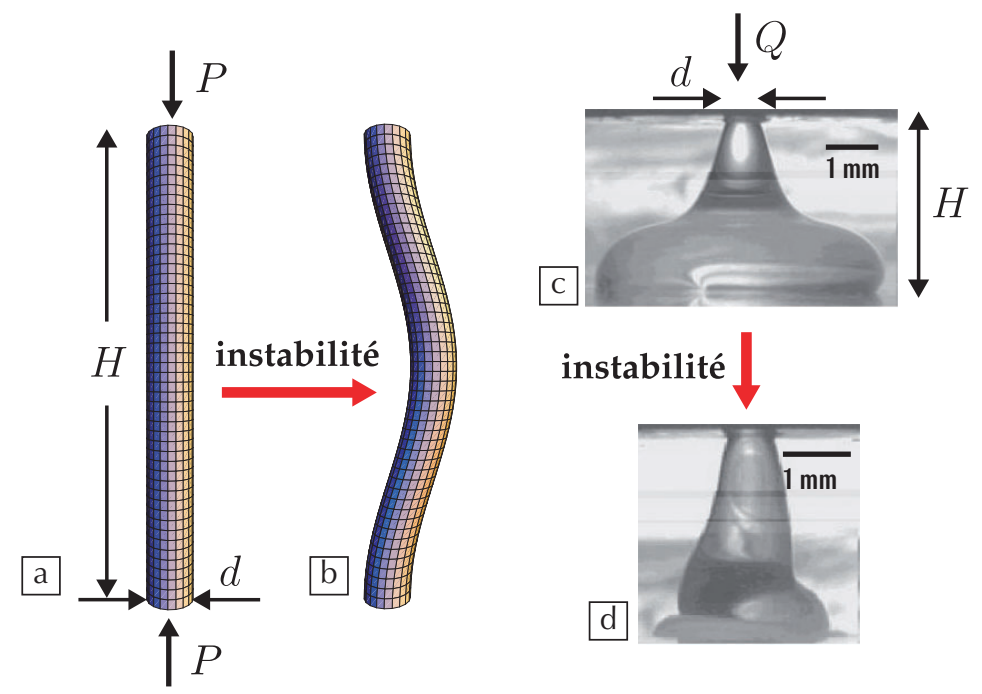

2. Démarrage des instabilités de flambage pour une tige élastique soumise à une force compressive (a, b) et pour une « tige » de fluide visqueux extrudée par un trou vers le bas (c, d).

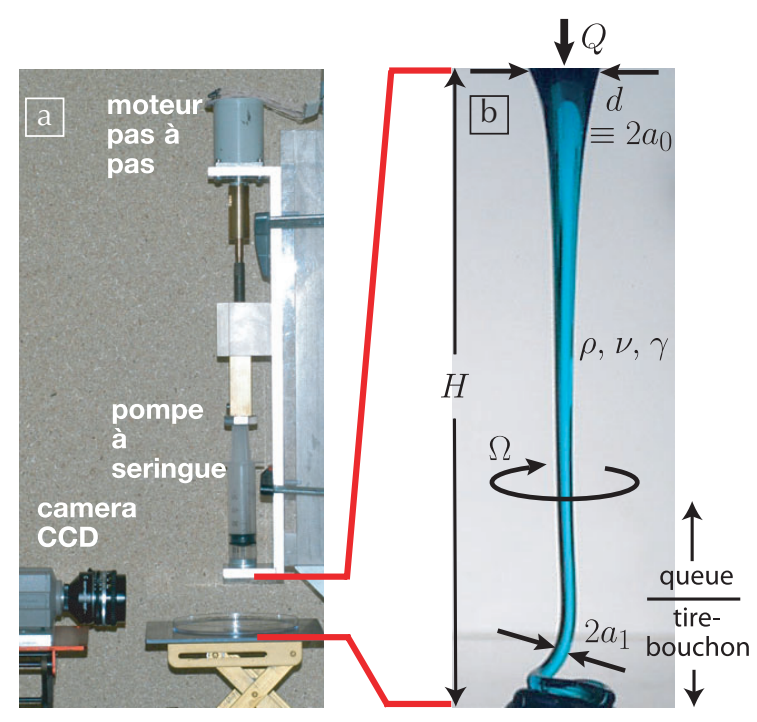

3. Dispositif expérimental pour étudier l'enroulement d'un filet de fluide visqueux (a) et définition des paramètres de l'enroulement (b). (à des facteurs numériques près) pour le démarrage du repliement d'une nappe.

Or, comprendre le démarrage des instabilités de flambage n'est qu'un début : il nous reste à explorer l'énorme domaine $H>H_{c}$ du flambage à amplitude finie. Dans ce dessein, nous avons choisi de focaliser notre attention sur le cas de l'enroulement d'un filet, plus simple à étudier (à la fois expérimentalement et numériquement) que le repliement d'une nappe.

\section{Mesures en laboratoire}

La figure 3 montre notre dispositif expérimental, où un mince filet d'huile de silicone $\left(\rho=0,97 \mathrm{~g} . \mathrm{cm}^{-3}, \gamma=21,5\right.$ dyne.cm $\left.{ }^{-1}\right)$, de viscosité $3 \times 10^{4}$ ou $10^{5}$ fois celle de l'eau, est extrudé de façon continue par une pompe à seringue. Cette figure montre aussi la forme typique du filet lorsque $H>>H_{c}$, avec une partie supérieure quasi verticale et fortement amincie vers le bas par la gravité (la «queue»), et une partie hélicoïdale (le "tire-bouchon ") tout en bas. Nous nous intéresserons surtout à la fréquence angulaire $\Omega$ de l'enroulement autour d'un axe vertical (fig. $3 b$ ).

La figure 4 présente une sélection de nos mesures de $\Omega$ en fonction de $H$ (symboles rouges). Elles sont d'une étonnante diversité. Si $H$ est faible, $\Omega$ décroît en fonction de $H$ (fig. 4a, $H<1 \mathrm{~cm}$ ). Par contre, si $H \sim$ plusieurs dizaines de $\mathrm{cm}, \Omega$ augmente rapidement en fonction de $H$ (fig. 4b). De plus, pour des valeurs intermédiaires de $H$ il peut y avoir des « trous " dans les courbes de mesures, où des fourchettes entières de valeurs de $\Omega$ sont absentes (fig. 4b, $\Omega \sim 15$ à $30 \mathrm{~s}^{-1}$ ). En fait, chaque trou correspond à une oscillation entre deux états métastables d'enroulement avec des fréquences différentes (fig. 4b, encart). Ce phénomène devient encore plus marqué si l'on augmente la viscosité ou diminue le taux d'extrusion $Q$ : on observe alors plusieurs trous entre des paliers quasi horizontaux, où se regroupent tous les points expérimentaux (fig. 4c).

Au premier abord donc, l'instabilité de l'enroulement semble receler tout et son contraire : diminution et augmentation de la fréquence avec la hauteur, variations continues et discontinues de la fréquence, comportements réguliers et irréguliers. Comment expliquer une telle diversité dans un seul phénomène?

\section{Une approche théorique}

Notre approche utilise un modèle numérique basé sur une théorie qui décrit le mouvement arbitraire d'un filet de fluide " mince ", dont le rayon est faible comparé au rayon de courbure de son axe. Cette théorie prend en compte quatre forces qui agissent sur le filet. Tout d'abord, il y a la gravité. Viennent ensuite les forces visqueuses qui résistent aux quatre modes de déformation possibles du filet : extension /compression axiales, flexion dans deux plans orthogonaux, et torsion. Troisièmement, les "forces " inertielles (centrifuge et de Coriolis) associées à la rotation du tirebouchon ainsi que l'accélération du fluide dans ce repère tournant deviennent importantes lorsque l'enroulement se produit à haute fréquence. La liste des forces est complétée par la capillarité qui a une influence significative (5 à 20\%), mais pas déterminante.

La forme finale de la théorie est un système d'équations d'ordre 17 qui décrit l'état stationnaire (dans le repère tournant) du filet, et que l'on peut résoudre numériquement [3]. Les lignes continues sur la figure 4 en montrent les prédictions, pour les mêmes valeurs de $v, \gamma, d$, et $Q$ que dans l'expérience de laboratoire correspondante. L'accord entre la théorie et les expériences est remarquable, d'autant plus que le modèle ne comporte aucun paramètre ajustable. Notre tâche maintenant est de comprendre ce que cet accord signifie en termes physiques.

\section{Quatre régimes d'enroulement}

Regardons d'abord la partie «tire-bouchon » du filet (fig. 3b), où la déformation est essentiellement de type flexural et torsionnel. Étant donné ces types de déformation, la théorie du filet "mince " nous permet de constater que trois différents types d'équilibre ("régimes ») entre les forces visqueuse, gravitationnelle et inertielle sont possibles. Dans le régime "visqueux " (V) d'abord, à faible hauteur $H$ et faible fréquence $\Omega$, la gravité et l'inertie sont négligeables devant les forces visqueuses. Ces dernières s'équilibrent donc toutes seules, comme dans de la pâte dentifrice qui sort lentement d'un tube sur une brosse à dents. Dans le régime « gravitationnel » $(G)$ ensuite, les forces visqueuses sont équilibrées par la gravité. Et lorsque l'enroulement se produit à très haute fréquence enfin, les forces visqueuses peuvent être contrebalancées par l'inertie dans un régime "inertiel » (I). Or, ces trois équilibres conduisent à trois lois d'échelle différentes pour la variation de la fréquence d'enroulement en fonction des paramètres expérimentaux [3-5] :

$$
\begin{aligned}
& \Omega_{\mathrm{V}}=\mathrm{Q} /\left(a_{1}^{2} H\right),
\end{aligned}
$$

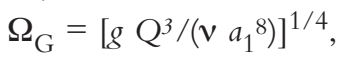

$$
\begin{aligned}
& \Omega_{\mathrm{I}}=\left[Q^{4 /(}\left(\begin{array}{ll}
\boldsymbol{v} & \left.a_{1}{ }^{10}\right)
\end{array}\right]^{1 / 3}\right. \text {. }
\end{aligned}
$$



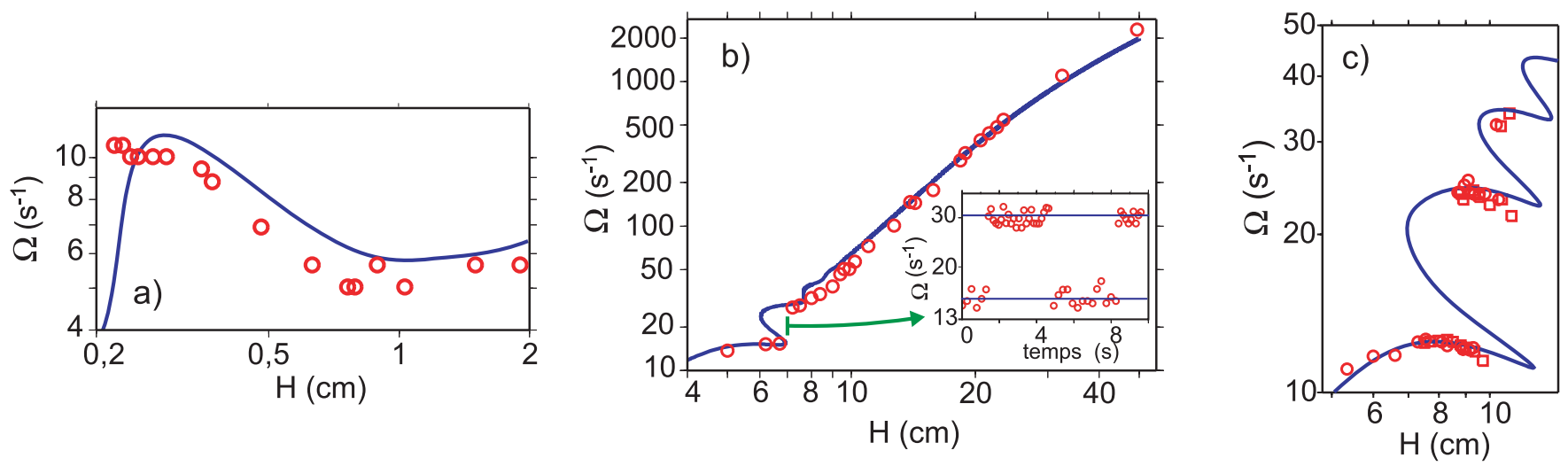

4. Fréquence d'enroulement en fonction de la hauteur de chute sous différentes conditions. Symboles rouges : mesures expérimentales ; lignes bleues : calculs numériques. (a) $d=0,068 \mathrm{~cm}, Q=0,0038 \mathrm{~cm}^{3} \mathrm{~s}^{-1}, v=1000 \mathrm{~cm}^{2} \mathrm{~s}^{-1}$. (b) Cas avec un trou et un débit beaucoup plus grands $\left(d=0,5 \mathrm{~cm}, Q=0,094 \mathrm{~cm}^{3} \mathrm{~s}^{-1}\right)$ et une viscosité plus faible $\left(v=300 \mathrm{~cm}^{2} \mathrm{~s}^{-1}\right)$. (c) comme (a), mais avec un débit plus faible $\left(Q=0,00215 \mathrm{~cm}^{3} \mathrm{~s}^{-1}\right)$. L'encart dans $(\mathrm{b})$ montre la fréquence en fonction du temps pour $H=7 \mathrm{~cm}$.

Notons enfin que ces trois expressions font intervenir le rayon $a_{1}$ du filet dans le tire-bouchon, qui dépend de l'ampleur de l'étirement gravitationnel de la "queue » du filet (fig. 3b). Le rayon $a_{1}$ est quasiment constant $\left(=a_{0}\right)$ dans le régime $\mathrm{V}$, mais il diminue avec la hauteur $H$ dans les régimes $G$ et I. En conséquence, la fréquence $\Omega_{\mathrm{V}}$ décroît en fonction de $H$, alors que $\Omega_{\mathrm{G}}$ et $\Omega_{\mathrm{I}}$ augmentent.

Jusqu'à présent, nous avons considéré la queue du filet comme un objet passif, dont la seule fonction serait de fixer $a_{1}$. Or, la queue peut aussi jouer un rôle actif, en exécutant des oscillations transverses libres qui correspondent à un quatrième régime « pendulaire » $(\mathrm{P})$. Les fréquences naturelles de ces oscillations sont toutes proportionnelles à la fréquence

$$
\Omega_{\mathrm{P}}=(g / H)^{1 / 2}
$$

d'un pendule simple, avec des constantes de proportionnalité qui dépendent de la distribution non uniforme de la masse du fluide (fig. 3b) le long de l'axe de la queue [6].

\section{l'assemblage du puzzle}

Les quatre lois d'échelle (1) et (2) reflètent tous les différents types d'équilibres de force possibles dans un filet qui s'enroule. Nous sommes donc en mesure d'assembler les morceaux du "puzzle " physique que nous avons déjà vu "en vrac" (fig. 4). Dans ce dessein, traçons (fig. 5) la variation théorique (calculée numériquement) de la fréquence $\Omega$ en fonction de la hauteur $H$ pour les paramètres de l'expérience de la figure 4c. En comparant les différentes parties de cette courbe avec nos lois d'échelle, on s'aperçoit que les quatre régimes d'enroulement $(\mathrm{V}, \mathrm{G}, \mathrm{P}$ et $\mathrm{I})$ se succèdent dans un ordre invariable lorsque la hauteur de chute augmente.

Pour $H<0,8 \mathrm{~cm}$, où l'enroulement se produit à basse fréquence, et la pesanteur et l'inertie du fluide sont négligeables devant les forces visqueuses, l'enroulement se produit donc en régime visqueux $(\mathrm{V})$, et la fréquence décroît avec la hauteur. L'image $V$ de la figure 5 montre à quel point le filet ressemble dans ce régime à de la pâte dentifrice. Pour $1 \mathrm{~cm}<H<7 \mathrm{~cm}$, la pesanteur du filet commence à se faire sentir, et le régime gravitationnel $(\mathrm{G})$ prend la relève. La fréquence augmente avec la hauteur, et le filet commence à développer la structure " queue + tire-bouchon " (fig. 5, image G). Pour $7 \mathrm{~cm}<H<14 \mathrm{~cm}$ ensuite, le filet entre dans le régime pendulaire $(\mathrm{P})$ où jusqu'à trois états d'enroulement de fréquences différentes sont possibles pour une seule et même hauteur de chute. C'est un régime de résonance, où les oscillations transverses de la queue sont excitées lorsqu'une de ses fréquences naturelles (lignes droites en tirets longs avec pente $-1 / 2$ ) est proche de la fréquence $\Omega$ fixée par le tire-bouchon. Les parties de la courbe en pointillés correspondent aux états qui sont instables par rapport à de petites perturbations et qui ne

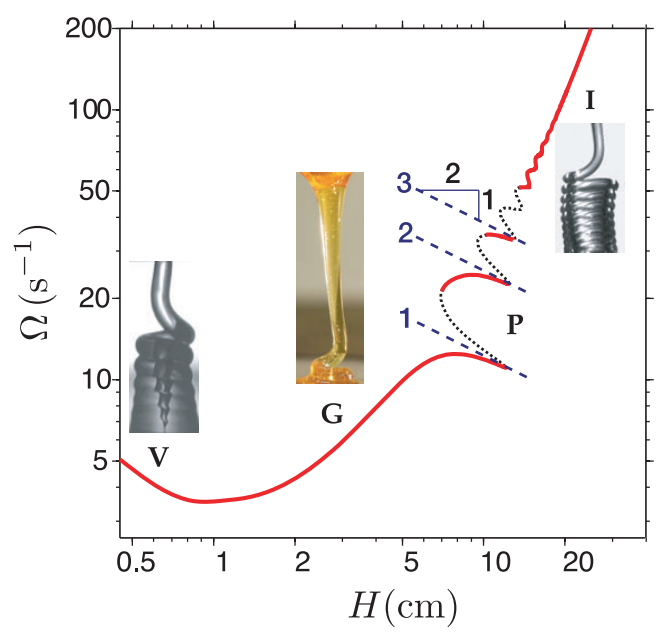

5. Vue d'ensemble des régimes d'enroulement V, G, P et I, sous la forme d'une courbe de la fréquence en fonction de la hauteur de chute pour les paramètres d'une expérience en laboratoire [6].

\section{Bibliographie}

1 - A. Bejan, "Buckling Flows: A New Frontier in Fluid Mechanics", Annual Review of Numerical Fluid Mechanics and Heat Transfer (1987), Vol. 1. Editors: T. C. Chawla, Hemisphere, Washington, DC, 262-304.

2 • B. Tchavdarov et al., "Buckling of thin liquid jets", Journal of Fluid Mechanics, 253 (1993), 593-615

3 - N.M. Ribe, "Coiling of viscous jets", Proceedings of the Royal Society of London, A460 (2004), 3223-3239.

$4 \cdot$ L. Mahadevan et al., "Correction: Fluid 'rope trick' investigated", Nature, 403 (2000), 502.

5 M. Maleki et al., "Liquid rope coiling on a solid surface", Physical Review Letters, 93 (2004), 214502

$6 \bullet$ N.M. Ribe et al.,"Multiple coexisting states of liquid rope coiling", Journal of Fluid Mechanics, 555 (2006), 275-297.

7 • L. Guillou-Frottier, J. Buttles, P. Olson, "Laboratory experiments on the structure of subducted lithosphere", Earth and Planetary Science Letters, 133 (1995) 19-34.

8 • N.M. Ribe, "Periodic folding of viscous sheets", Physical Review E, 68 (2003) 036305.

9 • N.M. Ribe et al.,"Buckling instabilities of subducted lithosphere beneath the transition zone", Earth and Planetary Science Letters, 254 (2007), 173-179. 

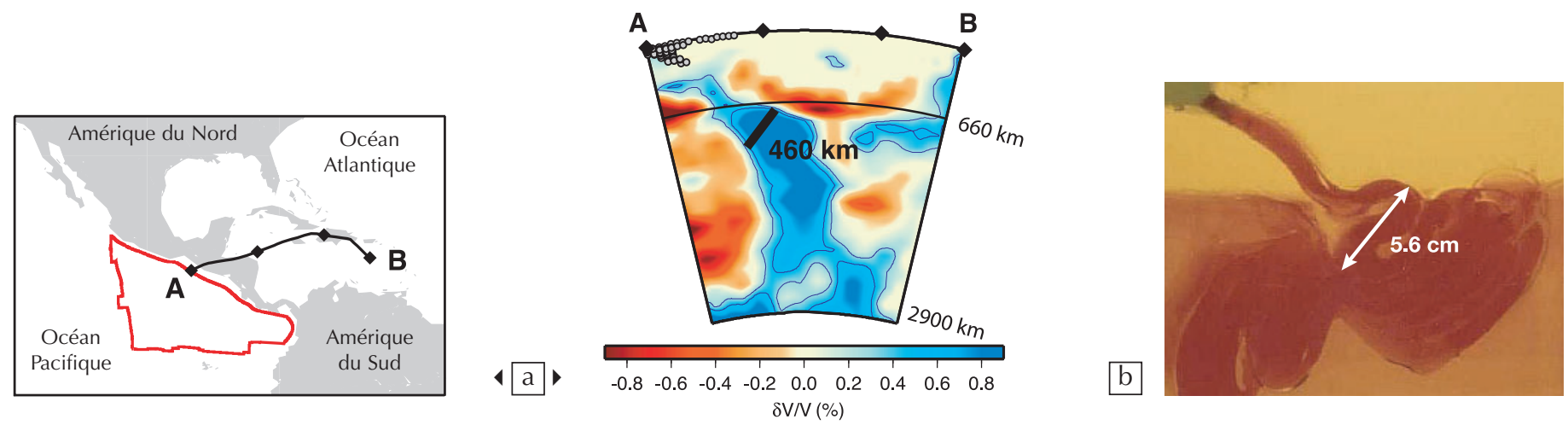

6. Repliement d'une plaque de lithosphère plongeant dans le manteau terrestre, et expérience analogique de laboratoire. (a) Coupe tomographique à travers le manteau sous l'Amérique centrale, montrant l'anomalie (bleue) de la vitesse des ondes élastiques de cisaillement qui correspond à la plaque subduite dans le passé. Les frontières actuelles de la plaque Cocos en surface sont indiquées en rouge sur la carte. (b) En laboratoire, une nappe de sirop de sucre très visqueux (rouge) injectée dans une cuve subit une instabilité de flambage à l'interface entre deux couches (orange et jaune) de sirop de sucre moins visqueux [7].

sont donc pas observables, ce qui explique les "trous" entre les paliers de mesures dans la figure $4 \mathrm{c}$. Enfin, pour $H>15 \mathrm{~cm}$, le filet entre dans le régime inertiel (I), où la fréquence augmente rapidement en fonction de la hauteur et où la structure en " tire-bouchon " est très bien développée.

\section{Une autre échelle: le manteau terrestre}

À des échelles d'espace et de temps beaucoup plus grandes, des instabilités de flambage peuvent se produire dans le manteau de notre planète. Il s'agit ici d'une géométrie différente - le repliement d'une nappe plutôt que l'enroulement d'un filet - mais la physique est la même que celle que l'on vient d'expliquer.

Le " manteau » est la partie de la Terre située entre le noyau de fer liquide $(2900 \mathrm{~km}$ de profondeur) et la croûte sous nos pieds (6 km d'épaisseur sous les océans). Bien que le manteau soit "solide " sur des échelles de temps courtes (e.g., celles de la propagation des ondes sismiques), il se déforme comme un fluide sur des échelles de temps géologiques. Cet écoulement prend la forme d'une convection très lente, dont le moteur est la variation de la densité des roches due aux variations de température et de composition chimique. La forte variation de la viscosité de ces roches avec la température fait que la partie supérieure froide du manteau est très forte mécaniquement : c'est la « lithosphère ", dont l'épaisseur maximum est de 80-100 km sous les océans. Par des mécanismes qui restent encore mal compris, elle se casse en gros morceaux (" plaques ") de 1000$10000 \mathrm{~km}$ de large, qui se déplacent latéralement et qui parfois replongent dans le manteau. Ce replongement s'appelle la «subduction".

Une question évidente concerne le sort réservé à la partie déjà subduite d'une plaque : jusqu'à quelle profondeur plonge-t-elle, et comment se déforme-t-elle en chemin ? Pour y répondre, il faut demander aux sismologues, qui savent cartographier la distribution tridimensionnelle des vitesses des ondes élastiques dans le manteau à partir d'enregistrements de tremblements de terre. Leur méthode, un peu analogue à la tomographie médicale mais à l'échelle du globe, consiste à examiner les temps de trajet des différentes ondes sismiques ayant traversé un volume donné du manteau pour savoir si leur vitesse y est plus rapide ou plus lente que la moyenne.

À titre d'exemple, la figure 6a montre une image tomographique d'une coupe verticale de la zone de subduction où la plaque Cocos plonge sous l'Amérique Centrale. Les couleurs bleues (orange) indiquent les endroits où la vitesse des ondes de cisaillement est plus rapide (lente) que la moyenne à cette profondeur, parce que la température y est plus basse (élevée). Or, vu qu'une plaque en subduction est plus froide que le manteau qui l'entoure, on peut conclure que l'anomalie bleue dans la figure 6a correspond à la partie de la plaque Cocos qui a été subduite dans le passé. Cette anomalie, plutôt mince dans le manteau supérieur $(<660 \mathrm{~km}$ de profondeur), s'épaissit rapidement ensuite pour atteindre une largeur de 600-700 km dans le manteau inférieur. Pourquoi ?

Une explication est suggérée par une expérience analogique ([7] ; fig. 6b). On remplit d'abord une cuve avec deux couches de sirop de sucre ayant des viscosités différentes, qui représentent les parties supérieure (moins visqueuse ; jaune) et inférieure (plus visqueuse; orange) du manteau. Ensuite, on injecte d'en haut une nappe de sirop de sucre encore plus visqueux (rouge). En rencontrant l'interface entre les deux couches de sirop, la nappe subit une instabilité de flambage, créant ainsi une masse de sirop plié dont la forme ressemble à celle de l'anomalie bleue dans la figure 6a. Pour vérifier que cette ressemblance n'est pas due au hasard, on fait appel à une loi d'échelle universelle pour l'amplitude $\delta \mathrm{du}$ flambage d'une nappe bidimensionnelle sans inertie en fonction de son épaisseur, sa viscosité, la vitesse d'injection et la hauteur de chute [8]. Pour l'expérience de la figure $6 \mathrm{~b}$, la loi prédit $\delta=5,7 \mathrm{~cm}$, en excellent accord avec l'amplitude mesurée $(5,6 \mathrm{~cm})$. Pour la subduction sous l'Amérique centrale, un calcul similaire donne $\delta=460 \mathrm{~km}$ ([9] ; fig. 6a, ligne noire), en bon accord avec la dimension de l'anomalie tomographique bleue. L'hypothèse d'une instabilité de flambage sous cette région semble donc raisonnable.

\section{Conclusion}

Les instabilités de flambage dans les fluides visqueux fascinent pour plusieurs raisons : la physique complexe qu'elles recèlent; leur apparition à des échelles très disparates, du laboratoire au manteau terrestre ; et leur beauté, tout simplement. Si leur dynamique est désormais comprise dans ses grandes lignes, il reste néanmoins plusieurs questions ouvertes. Côté géophysique, nous étudions actuellement comment la présence d'un fluide visqueux ambiant (le " manteau ") influence le flambage d'une nappe ; et nous attendons avec impatience le jour où la tomographie sismique aura une résolution suffisante pour vérifier la présence des "plis » individuels dans les plaques subduites. Quant à l'enroulement, nos expériences récentes ne cessent de révéler des surprises : instabilités secondaires du tire-bouchon, ondes spirales de bulles d'air piégées, oscillations entre enroulement et repliement d'un filet, cessation hystérétique de l'enroulement à de très grandes hauteurs de chute... Autant de phénomènes qui restent à expliquer. Affaire à suivre.. 